\title{
Expression of Nerve-Muscle Topography During Development
}

\author{
Michael B. Laskowski and Julie A. High \\ Department of Physiology, St. Louis University School of Medicine, St. Louis, Missouri 63104
}

\begin{abstract}
Previous studies have indicated that in 2 muscles of the adult rat, the anterior serratus and the diaphragm, the rostrocaudal axis of the motoneuron pool projects topographically onto the rostrocaudal axis of the muscle. In the present work we have asked whether this orderly topography emerges as a function of postnatal synaptic rearrangment or whether this pattern is already established at birth. The anterior serratus muscle was studied over the period ranging from embryonic day 17 through postnatal day 30 . Using 2 criteria of topography, average segmental innervation and average target field of cervical roots $C_{6}$ and $C_{7}$, we found that a topographic distribution of the motoneuron pool is already present prior to birth and maintained throughout the postnatal period. Moreover, both $C_{8}$ and $C_{7}$ form an orderly map over the surface of the serratus in the embryo, and the topography is sharpened during postnatal periods. The diaphragm also is topographically innervated at birth and undergoes a comparable sharpening of the projection map postnatally. We conclude that the topographic projection of motoneurons is established prior to birth in these muscles, and postnatal synaptic rearrangement serves to sharpen the topographic map toward the adult pattern. These results also suggest that the pursuit of basic mechanisms underlying topography should be directed toward initial embryonic nerve-muscle contacts.
\end{abstract}

Motoneuron pools project onto muscles in a topographic manner (Sherrington, 1892; Browne, 1950). In its broader meaning, topography refers to the ordered projection of consecutive ventral roots onto groups of muscles associated with a limb or the trunk. Most previous studies of topography have centered on groups of limb muscles, but more recently attention has focused on the topographic projection of neurons onto a single muscle (Swett et al., 1970; English and Letbetter, 1982a, b; Brown and Booth, 1983a, b; Bennett and Lavidis, 1984a, b; English and Weeks, 1984; Laskowski and Sanes, 1987a; Balice-Gordon and Thompson, 1988a, b; Bennett and Ho, 1988). When a single muscle is considered, topography has a more restricted meaning. In this context, topography means that the motoneuron pool maps systematically over the surface of a muscle or its compartments (Weeks and English, 1985; Laskowski and Sanes,

Received Jan. 25, 1988; revised May 16, 1988; accepted May 20, 1988

We thank Melody Mance for assistance and J. Sanes and D. Purves for comments on the manuscript. We also thank J. Sanes for helpful suggestions during the course of these studies. This work was supported by the Muscular Dystrophy Association of America.

Correspondence should be addressed to Michael B. Laskowski, Wami Medical Program, University of Idaho, Moscow, ID 83843.

Copyright (c) 1989 Society for Neuroscience $0270-6474 / 89 / 010175-08 \$ 02.00 / 0$ 1987a). If the muscle is compartmentalized, then there is an ordered mapping of motoneurons within those compartments (English and Letbetter, 1982a, b; Bennett and Ho, 1988). In this map, each ventral root has a progressively increasing or decreasing influence over the muscle surface, depending on the position of its motoneurons within the respective motor nucleus. In our effort to define the mapping of motoneuron pools onto a single muscle, we selected the anterior serratus and diaphragm. Both muscles have served as useful models for exploring topography. In adults we observed an orderly topographic mapping of motoneuron pools onto muscle targets; the rostrocaudal organization of the motoneuron pool mapped onto the rostrocaudal axis of both muscles (Laskowski and Sanes, 1987a). This map is restored, albeit imperfectly, when axotomized motoneurons reinnervate their muscle targets (Laskowski and Sanes, 1987b, 1988). Moreover, regenerating neonatal motoneurons reinnervate their muscle targets even more selectively (Laskowski and Sanes, 1988). In the present study we asked whether this topographic map is established prior to birth or whether the synaptic rearrangement that occurs postnatally is primarily responsible.

It is well established that at birth mammalian muscle fibers are multiply innervated and that during the first few weeks of postnatal development synaptic rearrangement takes place (Redfern, 1970; Bennett and Pettigrew, 1974; see review by Bennett, 1983). By 3 weeks of age most endplates contain the terminal of a single axon. Two alternative hypotheses emerge. First, growing neurites might contact their targets in a relatively random pattern. Topography would then emerge from the selective loss of inappropriate terminals during the period of synaptic rearrangement. This alternative places a major emphasis on postnatal mechanisms and reduces the importance of growing neurites making contact with matched synaptic partners. In its favor, Bennett and Lavidis (1984a, b) reported that some limb muscles receive innervation in a generally uniform projection; thereafter, through the process of selective removal of innervation by one root, the territory innervated by any spinal nerve is selectively modified. What emerges is a topographic pattern of innervation.

Alternatively, growing neurites may select their appropriate targets upon first contact with muscle cells. This would mean that topography is already present prior to birth and reduces the role of synaptic rearrangment to that of refining the topography already established. Brown and Booth (1983a, b) observed that already at birth a small but significant topography was present in the rat gluteus muscle. This positional gradient was greatly sharpened during the period of postnatal synaptic rearrangement. A similar pattern is seen in the autonomic nervous system. The basic topographic innervation is present at birth, and postnatal synaptic rearrangement sharpens this topography 

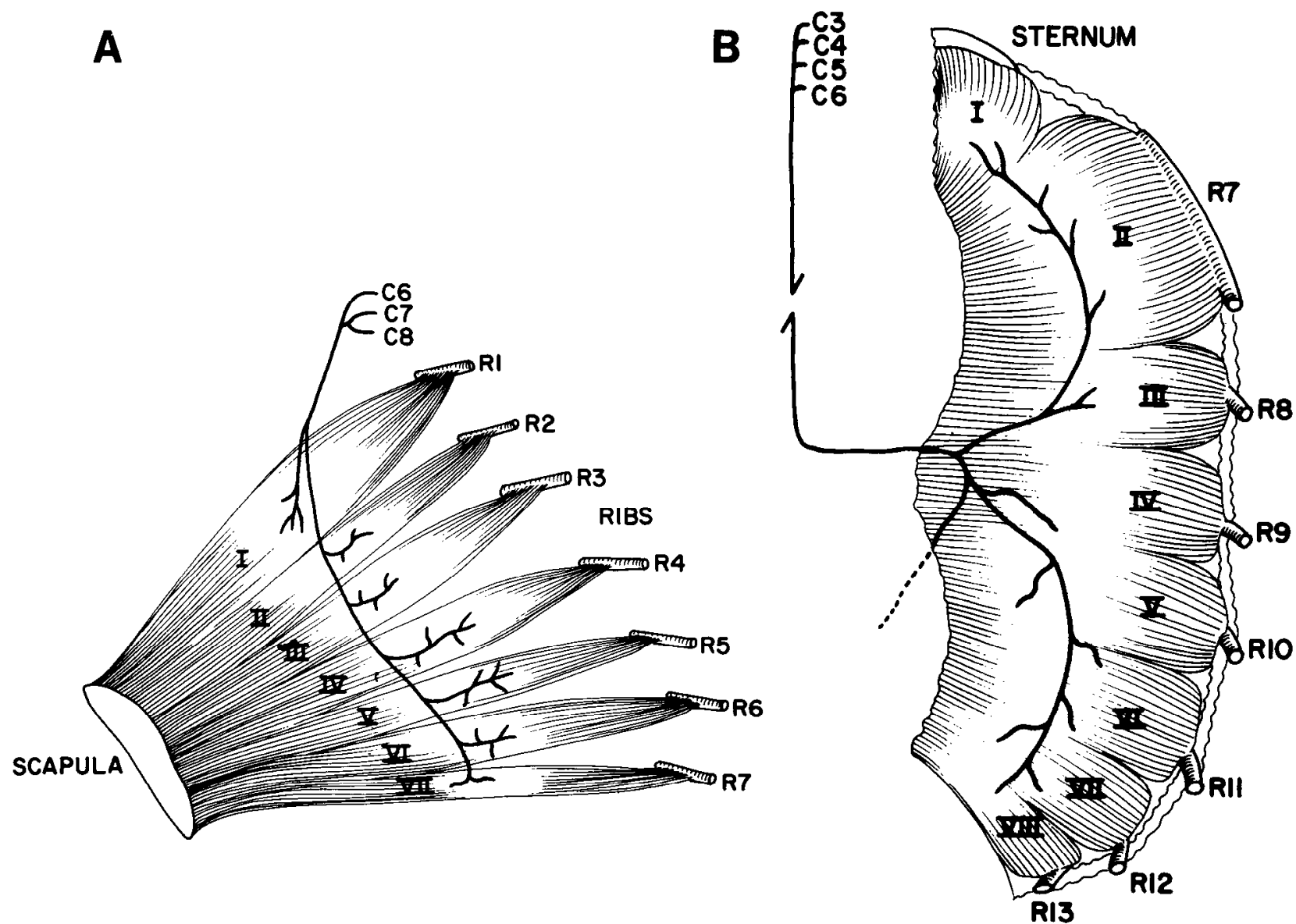

Figure 1. Drawing of muscles used in this study. A, The anterior serratus consists of 7 sectors formed by the origin of muscle fibers from ribs 17. The long thoracic nerve, which arises from $\mathrm{C} 6-\mathrm{C} 8$, innervates the scrratus. $B$, The hemidiaphragm consists of 8 sectors formed by the origin of its fibers from connective tissue associated with the sternum and with ribs $7-13$. Innervation is provided by the phrenic nerve, which arises from ventral roots $\mathrm{C} 3-\mathrm{C} 6$. Drawings are not to scale.

(Lichtman and Purves, 1980). Here, we sought to resolve this question in the somatic motor system by studying the muscles of the rat throughout the period beginning in late embryonic development and extending through the first month of postnatal life. We selected the diaphragm and anterior serratus muscles because wc had mapped the topography in adults and had demonstrated selective reinnervation (Laskowski and Sanes, 1987a, b, 1988). Our results show that while the map may be sharpened by postnatal events, it is clearly present before birth. This allows us to focus our attention on embryonic development in the pursuit of the fundamental processes responsible for topography.

\section{Materials and Methods}

Dissection. We used timed, pregnant rats of the Sprague-Dawley strain for this study. For experiments on embryonic muscles, on the designated day of gestation (E17 or E19), the pregnant rat was anesthetized with chloral hydrate $(0.2 \mathrm{gm} / \mathrm{kg}$, i.p.) and the fetus was removed. Anesthesia of the fetus was achieved by cooling on ice. Dissection began after all movement ceased. Litters were typically delivered on day 22 of gestation, which was postnatal day zero (P0). We studied muscles at 5 designated postnatal ages, P2, P7, P14, P20, and P30 and at 2 embryonic ages, E17 and E19. In practice, each of these designated postnatal ages is the average of a narrow range: $\mathrm{P} 2$ (range, $\mathrm{P0}-3$ ), $\mathrm{P} 7$ (P6-8), P14 (P1216), P20 (P19-21), P30 (P28-32). Pups up to $7 \mathrm{~d}$ old were anesthetized on ice; older pups were anesthetized with chloral hydrate $(0.1 \mathrm{gm} / \mathrm{kg})$. All neonates were perfused through the left ventricle to remove the blood and thus simplify later dissection.
The diaphragm and anterior serratus muscles together with their respective ventral roots were dissected. We selected these muscles for several reasons. First, they are relatively thin and flat and are subdivided into several easily identifiable sectors even in the embryo (Fig. 1). Second, they are trunk muscles whose rostrocaudal axis of orientation parallels that of the spinal cord. Third, they are innervated by more than one ventral root, a feature that is necessary in order to define topography. Finally, we have studied these muscles in adult animals and have established an orderly topographic projection of motoneuron pools on to these muscles (Laskowski and Sanes, 1987a). For the anterior serratus we isolated cervical ventral roots $C_{6}, C_{7}$, and $C_{8}$ in a manner similar to that used for adults (Laskowski and Sanes, 1987a). In this procedure, which we used for all postnatal preparations, the ventral roots were dissected away from the vertebral column. The muscle with its attached nerve and roots was pinned in a Sylgard-coated petri dish. In embryonic preparations, ventral roots were dissected away from the spinal cord but left within the vertebral column. Most of the tissue surrounding the cervical portion of the column was dissected away, and the muscle together with the vertebral column and thoracic nerve were pinned to the petri dish. In these embryos we found that if sufficient tissue was dissected away from the vertebral column and nerve, the preparation remained viable for several hours. The diaphragm was studied at postnatal ages $\mathrm{P} 0-\mathrm{P} 3$. Cervical ventral roots $\mathrm{C}_{3}, \mathrm{C}_{4}, \mathrm{C}_{5}$, and $\mathrm{C}_{6}$ wcre dissccted away from the vertebral column, and the diaphragm together with the phrenic nerve and its ventral roots was pinned in the petri dish. Because the adult studies of topography were carried out on muscles of the right side, we used only muscles of the right side in these experiments. Muscle-nerve preparations kept at room temperature were superperfused with oxygenated Ringer's solution containing $144 \mathrm{~mm}$ $\mathrm{NaCl}, 4 \mathrm{~mm} \mathrm{KCl}, 1 \mathrm{mM} \mathrm{MgCl} 2,1 \mathrm{mM} \mathrm{KH} \mathrm{KH}_{4}, 4 \mathrm{~mm}$ HEPES buffer, $11 \mathrm{~mm}$ glucose, and $5 \mathrm{~mm} \mathrm{CaCl}_{2}, \mathrm{pH} 7.3$. 

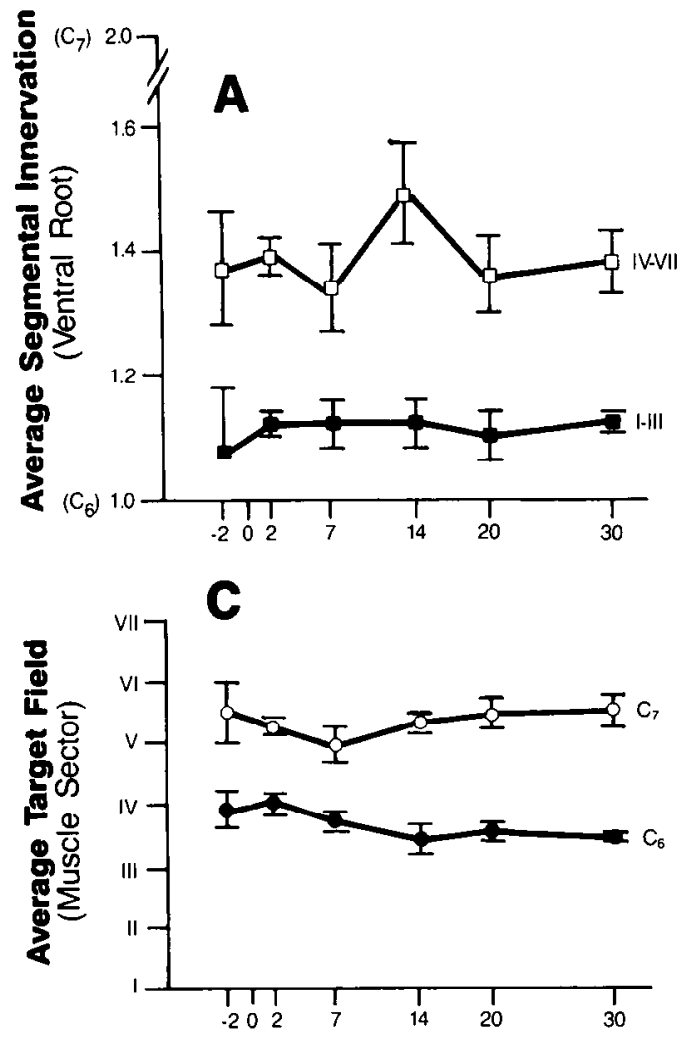

Age (Days)
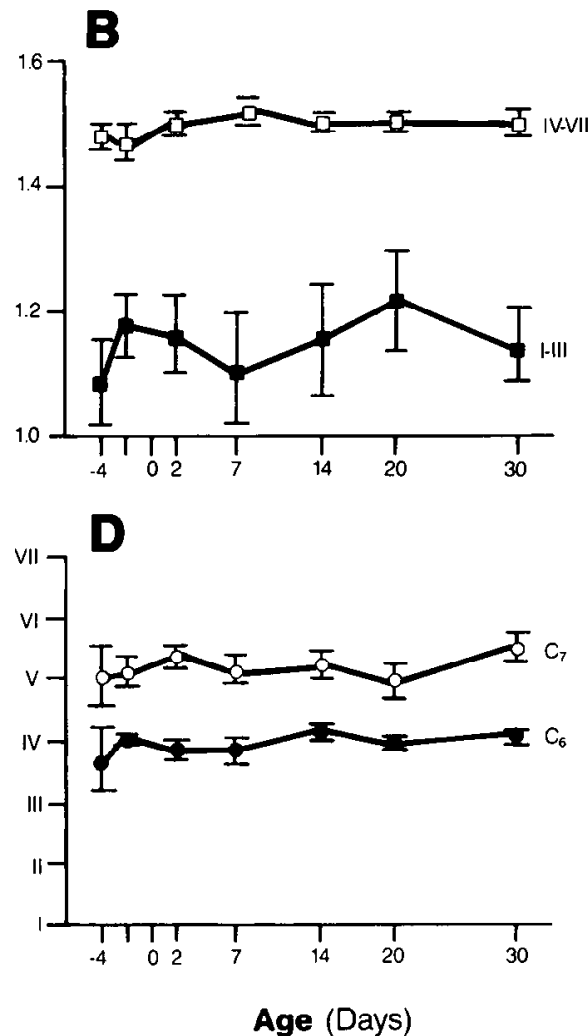

Figure 2. Topographically specific innervation of the developing anterior serratus muscle. $A$ and $C$ were obtained from synaptic potentials; $B$ and $D$ were obtained from contractile responses. The average segmental innervation of each half of the muscle and the average target field of each ventral root were calculated as described in Results, based on synaptic potentials recorded intracellularly and on localized contractions rated visually. Ages marked -2 and -4 represent $E 19$ and E17, respectively, and age $O$ indicates day of birth. Values are means \pm SE of data from 3-10 muscles at each age (average of 6 muscles). In each muscle, 10 endplates were sampled from each of 7 sectors. At each age group in $A$ and $B$ average segmental innervation of sectors I-III (closed sym$b o l s$ ) is significantly different from that of IV-VII (open symbols) at the $p<$ 0.05 level, using the Student's $t$ test. Similarly in $C$ and $D$, the average target innervation by $\mathrm{C}_{6}$ (closed symbol) and $\mathrm{C}_{7}$ (open symbol) is different at all age groups at the $p<0.05$ level.
Electrophysiology. Each root was placed in a suction electrode, and square-wave pulses of $1-10 \mathrm{~V}$ at 0.1 msec duration were delivered to each root in sequence. The relative vigor of contractions evoked in each muscle sector (see Fig. $1, A, B$ ) by these stimuli was rated on a scale of $0-4$, with 4 being the strongest response. Threshold for visible contractile responses was typically $1-3 \mathrm{~V}$ and did not change significantly over the course of the experiment. Voltage was gradually increased until a maximal contractile response was achieved, and a voltage of at least twice this maximum was used for all subsequent procedures. While visual estimates of contractile force are admittedly less accurate than measured tension, they have served as reliable estimates of the spatial distribution of any given ventral root onto a muscle in 2 ways. First, in previous studies of topography in normal (Laskowski and Sanes, 1987a) and reinnervated (Laskowski and Sanes, 1987b, 1988) muscles, maps derived from contractile responses were highly correlated with maps derived from intracellular synaptic potentials. Second, in any given muscle sector, the contractile force estimated visually by stimulation of a ventral root corresponds to the proportion of synaptic inputs to that sector. Consequently, where possible both synaptic potentials and contractile responses were assessed in these experiments.

For intracellular studies, muscles were stretched to prevent displacement of the microelectrode. Glass micropipettes filled with $3 \mathrm{M} \mathrm{KCl}$ or $2 \mathrm{M} \mathrm{KAc}$ were inserted into muscle cells at the endplate zone, and each root was stimulated in sequence. Only surface fibers were impaled to avoid the possibility of an electrical coupling artifact occasionally seen when recording from deeper layers (Soha et al., 1987). We learned that stretching embryonic or early neonatal muscles causes depolarization of muscle membranes, whereas no such effect is seen in muscles older than P7. In older animals where resting membrane potential was at least $-60 \mathrm{mV}$, an action potential usually obscured the endplate potential. The experimental protocol was as follows. Each relevant ventral root was stimulated in turn. When a synaptic potential was recorded, the root or roots providing input were noted. Ten cells were sampled in this way from each sector in every muscle. Thus, the overall pattern of topographic input from each root could be estimated. These data were used to provide 2 estimates of topography, i.e., synaptic input and contractile responses as described in detail in Results.

\section{Results}

Topography in the developing anterior serratus

In adult rats, the rostrocaudal axis of the motoneuron pool is projected systematically onto the rostrocaudal axis of the anterior serratus muscle. Thus, rostral muscle sectors are innervated by rostral portions of the motor pool, whereas caudal muscle sectors receive more caudally derived motoneurons. We asked whether the same selectivity was apparent in late embryonic (E17 and E19) and postnatal (P2, P7, P14, P20, and P30) periods. We documented this selectivity in 2 different ways.

First, we calculated an index reflecting the average segmental innervation of each half of the anterior serratus muscle, i.e., muscle sectors I-III (rostral half) versus sectors IV-VII (caudal half). We selected this particular subdivision for 2 reasons. First, anatomically the rostral 3 sectors are separated from the caudal 4 sectors by the passage of the pectoralis minor from its origin on the ribs. These lower 4 sectors fuse at their insertion onto the scapula. Second, preliminary studies indicated that the territory innervated by $C_{7}$ was usually restricted to sectors IV-VII. Because embryonic ventral roots are too small and delicate to subdivide in a consistent manner (as is possible in adults), wc stimulated the entire ventral root and compared responses to $C_{6}$ versus $C_{7} ; C_{6}$ was assigned the number $1, C$, the number 2 . (As in adults, $\mathrm{C}_{8}$ does not provide motor innervation to the anterior serratus in neonates.) For example, we recorded 29 inputs from $\mathrm{C}_{6}$ and 1 input from $\mathrm{C}_{7}$ in the rostral half (sectors I-III) of one muscle at $\mathrm{P} 30$. This half muscle received a score of $[29(1)+1(2)] /(29+1)=1.03$. Thus, by this measure muscle sectors innervated by more caudally derived subsets of axons 
Figure 3. Topographic mapping of individual ventral roots onto anterior serratus muscles at 4 different ages. Closed symbols, $\mathrm{C}_{6}$; open symbols, $\mathrm{C}_{7}$. Each point represents the mean \pm SE of synaptic potentials recorded in 4-10 muscles at a particular age. Data from embryonic muscles were pooled; 1 at E17 and 3 at E19. In each muscle, 10 endplates were sampled, from each of 7 sectors and the percentage innervation by either $\mathrm{C}_{6}$ or $\mathrm{C}_{7}$ was calculated. At each age shown the sum of the percentage innervation by $\mathrm{C}_{6}$ and $\mathrm{C}_{7}$ will equal $100 \%$. This is because the number of inputs in each sector by either $C_{6}$ or $\mathrm{C}_{\text {, was divided by the total number }}$ of inputs to that sector by both roots. This scheme was chosen because it incorporates the overall influence of each root in the calculation and allows comparison between ages, as some terminals are withdrawn.
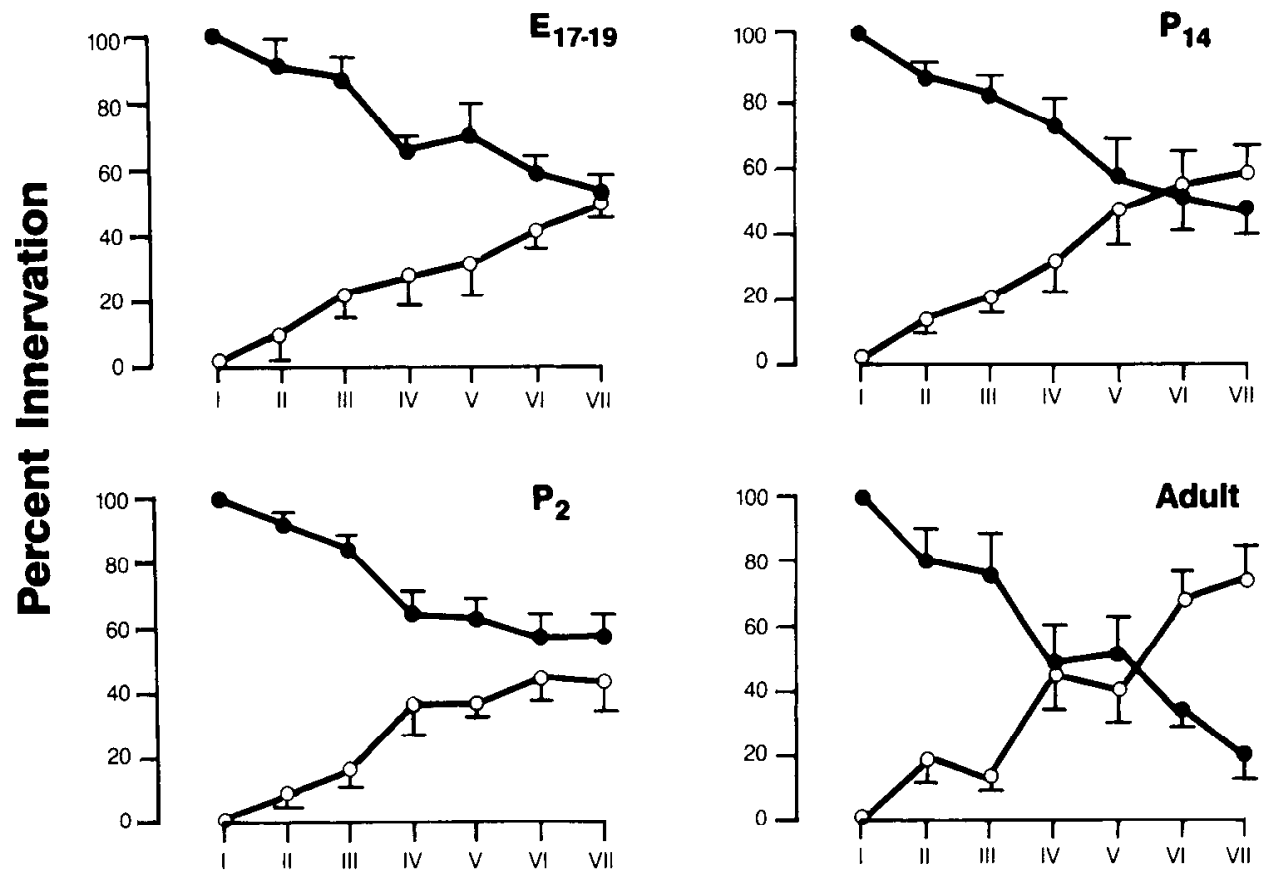

Muscle Sector

receive progressively higher segmental innervation numbers. In this same muscle, for example, the caudal half (sectors IV-VII) received 28 inputs from $C_{6}$ and 15 from $C_{7}$. This half muscle reccived a score of $[28(1)+15(2)] /(28+15)-1.35$. A similar index of average segmental innervation was used to summarize local contractile responses. Only strong $(3+$ or $4+)$, visually estimated contractile responses were used in the evaluation. We were particularly interested in the major innervation of muscle sectors by any one root. Thus, we reported only strong $(3+$ or $4+$ ) contractile responses. If we were to include all contractile responses regardless of strength, the results would be fundamentally the same as reported here. The results of experiments wherein we measured synaptic potentials and localized contractile responses from muscles at E17-P30 are illustrated in Figure 2. When synaptic potentials are considered (Fig. 2A), rostral muscle sectors receive innervation from more rostral portions of the motor pool than caudal muscle sectors. Because of technical difficulties we were able to record synaptic potentials from only one muscle at age E17. Even in this muscle, however, average segmental innervation of rostral sectors was 1.0 , whereas innervation to caudal sectors was 1.33. A similar pattern emerged when we analyzed average segmental innervation assessed by localized contractile responses (Fig. $2 B$ ). At each stage of development, caudal muscle sectors receive, on average, more caudal innervation than rostral muscle sectors. We were able to examine contractile responses in 3 muscle preparations at E17 and found the same topographic pattern. These data indicate that by 2 criteria, synaptic potentials and contractile responses, topographic order is present at all ages studied, including embryonic.

A second way of analyzing the data is to calculate the center area of muscle that is innervated by each ventral root, that is the average target field of each root. For example, for the P30 muscle considered above in response to stimulation of the $C_{7}$ root, we recorded synaptic potentials from 1 muscle fiber in sector II, 2 from sector IV, 4 from sector V, 5 from sector VI, and 4 from sector VII. In this muscle, the $C_{7}$ root was scored as having an average target field of $[1(2)+2(4)+4(5)+5(6)$ $+4(7)] /(1+2+4+5+4)=5.5$. Such indices were calculated from measurements of synaptic potentials or localized contractile responses and are illustrated in Figure 2, $C$ and $D$, respectively. Based upon synaptic potentials, the average target field of $\mathrm{C}_{7}$ is significantly more caudal than that of $\mathrm{C}_{6}$ at all ages from E19 through P30. Data based upon contractile responses (Fig. $2 D$ ) confirm this and extend this difference in target field for each root to $\mathrm{E} 17$ embryos. This analysis confirms our conclusion from the average segmental innervation of muscle that motoneuron pools project onto muscles topographically throughout the developmental period under consideration.

\section{Mapping of ventral roots onto serratus muscles}

The data upon which Figure 2 is based were analyzed from another perspective. We asked whether there was a difference in the mapping of individual roots onto muscles at various developmental ages. This would indicate how each root is distributed over the surface of a muscle at each developmental stage. Figure 3 illustrates such an analysis and compares this with the adult pattern recalculated from data presented by Laskowski and Sanes (1987a). At each stage (E17-E19, P2, and Pl4) both $C_{6}$ and $C_{7}$ ventral roots formed a rostrocaudal map over the muscle surface. Three important features emerge from the analysis illustratcd in Figure 3. First, already in cmbryonic serratus muscles there is a striking difference in innervation pattern in rostral sectors. This pattern once established in the embryo does not change significantly through the adult period. Second, at each age the influence of $\mathrm{C}_{6}$ gradually diminishes over progressively more caudal sectors, while that of $\mathrm{C}_{7}$ is steadily augmented. This pattern of ordered mapping is evident in embryonic muscles and continues throughout development. Third, while $\mathrm{C}_{7}$ has a greater influence on caudal than rostral 


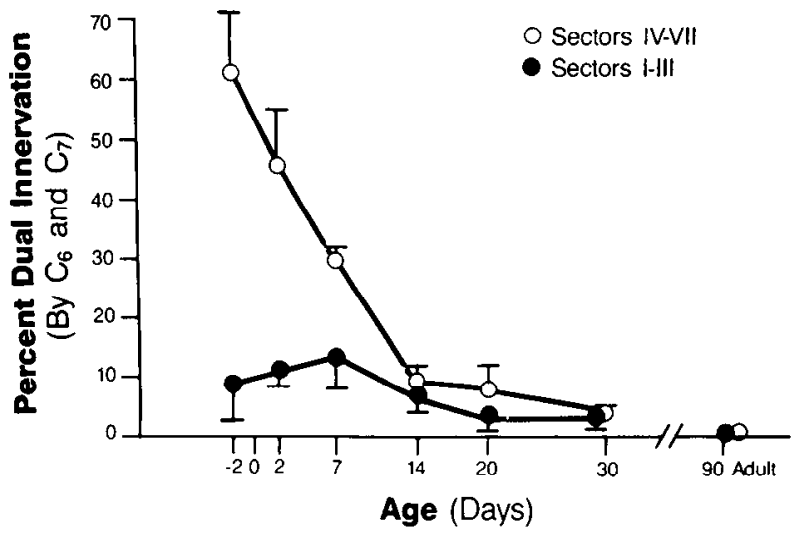

Figure 4. Percentage of muscle fibers innervated by both ventral roots $\mathrm{C}_{6}$ and $\mathrm{C}_{7}$ as a function of age. Each point represents the mean $\pm \mathrm{SE}$ of synaptic potentials recorded in 4-10 muscles at each age. Open circles represent the caudal half of the serratus and closed circles the rostral half.

sectors throughout the periods studied, it eventually emerges as the dominant root in sectors VI and VII after the second week of postnatal development (P14). These data indicate, therefore, that an ordered topography is already established in the embryo and that the pattern is sharpened during the postnatal period to resemble more closely the adult pattern.

Dual innervation of serratus muscle fibers by $C_{6}$ and $C_{7}$

In the course of our experiments we noted that some endplates were innervated by both $\mathrm{C}_{6}$ and $\mathrm{C}_{7}$ and that this dual innervation changed during development. We calculated the percentage of endplates that received input from both $\mathrm{C}_{6}$ and $\mathrm{C}_{7}$ for each developmental age and plotted them as shown in Figure 4. We found that the majority $(61 \%)$ of endplates in caudal sectors (IV-VII) of E19 muscles were innervated by both roots, and this gradually diminished during postnatal development. Only $9 \%$ of rostral sectors were so innervated, and this dual innervation pattern also diminished over the next 4 weeks. Two conclusions can be drawn from this analysis. First, like other muscles studied, synapse elimination does occur in the serratus. Second, muscle fibers in caudal sectors are more likely to be innervated by both roots, and this gradually diminishes with postnatal development.

\section{Topography in the developing diaphragm}

In order to confirm our observations in another muscle, we selected the diaphragm primarily because we had characterized its adult topography, but also because its postnatal synaptic rearrangement has been described and is essentially complete by P14 (Redfern, 1970; Bennett and Pettigrew, 1974). The analysis compared rostral (sectors I-III) versus caudal (sectors IVVIII) halves of the diaphragm because these are the territories served by the primary branches of the phrenic nerve (Laskowski and Sanes, 1987a). Figure 5 illustrates the results from diaphragms at ages $\mathrm{P} 0-\mathrm{P} 3$. Using the estimate of average segmental innervation based upon recordings of synaptic potentials, we observed that already at this early postnatal age, rostral muscle sectors (I-III) are innervated by more rostral spinal cord segments, and caudal muscle sectors (IV-VIII) are innervated by more caudal elements of the motor pool. Calculations based on contractile responses confirm this. When the average target field of each ventral root $\left(\mathrm{C}_{3}, \mathrm{C}_{4}, \mathrm{C}_{5}\right.$, and $\left.\mathrm{C}_{6}\right)$ was estimated, we found
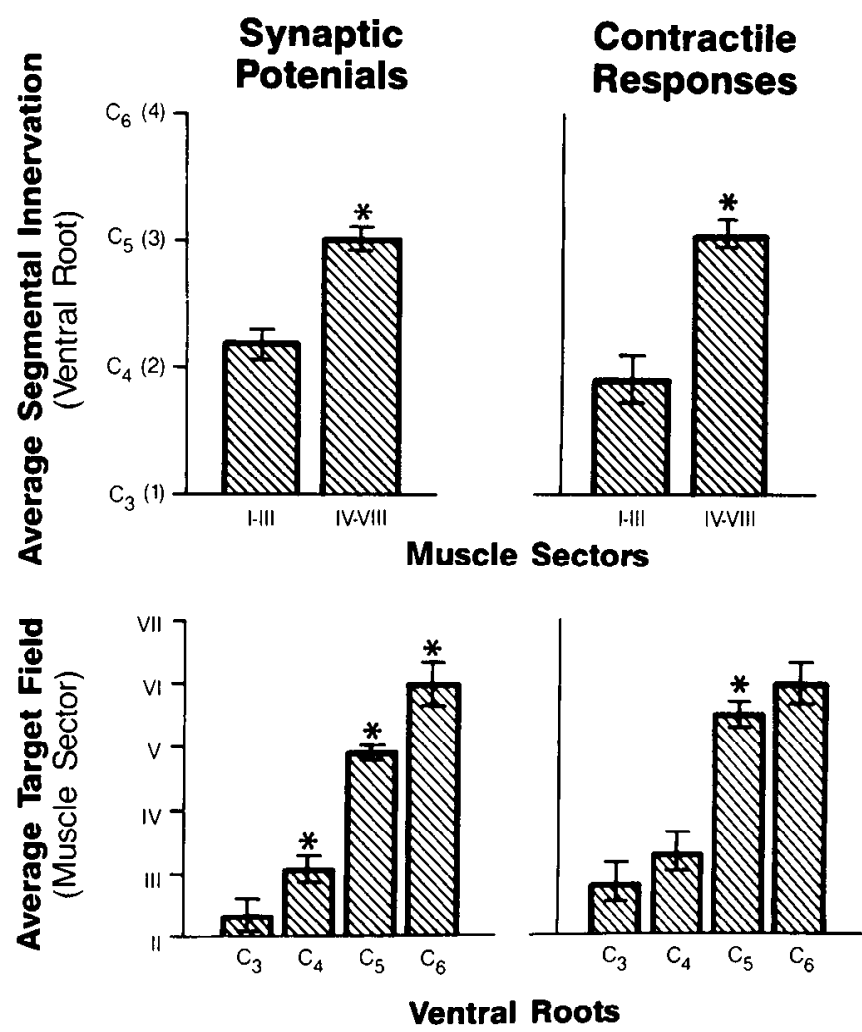

Figure 5. Topographically specific innervation of the early postnatal diaphragm (P0-P3). Average segmental innervation was calculated as described in Results. Asterisk denotes significant difference from the more rostral half of the diaphragm at the $p<0.05$ level (Student's $t$ test). Average target field illustrates the average muscle sector that each ventral root innervates. The asterisk here denotes significant difference from the immediately more rostral ventral root $(p<0.05)$. Data are expressed as the means \pm SE from 4 preparations.

that progressively more caudal roots projected onto progressively more caudal muscle sectors. This conclusion, based on synaptic potentials, was confirmed by estimating contractile responses of individual sectors. These observations indicate that, like the serratus, the topography of the diaphragm is evident at early postnatal times (PO-P3).

\section{Mapping of individual ventral roots onto the diaphragm}

We analyzed the mapping of each of the 4 ventral roots over the surface of the diaphragm (Fig. 6) and compared our results from neonates with results from our previous report (Laskowski and Sanes, 1987a). Figure 6 illustrates 3 main observations. First, shortly after birth (P0-P3) each root projects over the surface of the diaphragm in a rostrocaudal order similar to the adult pattern. Thus, $\mathrm{C}_{4}$ favors more rostral sectors, whereas $\mathrm{C}_{6}$ favors more caudal sectors, as seen in adults. Second, in neonates $\mathrm{C}_{3}$ innervates rostral sectors exclusively, and its influence is all but lost in adults. Third, the topographic map is sharpened between birth and adulthood, such that $\mathrm{C}_{4}$ comes to have greater influence in rostral sectors and $\mathrm{C}_{6}$ in caudal sectors. Shortly after birth, $65 \%$ of diaphragm muscle fibers are innervated by more than one of the cervical roots, a redundancy that disappears by adulthood (data not shown). These observations indicate that already within the first few days after birth an orderly map is established over the surface of the diaphragm. As with the anterior serratus, this map is not fundamentally different from the 

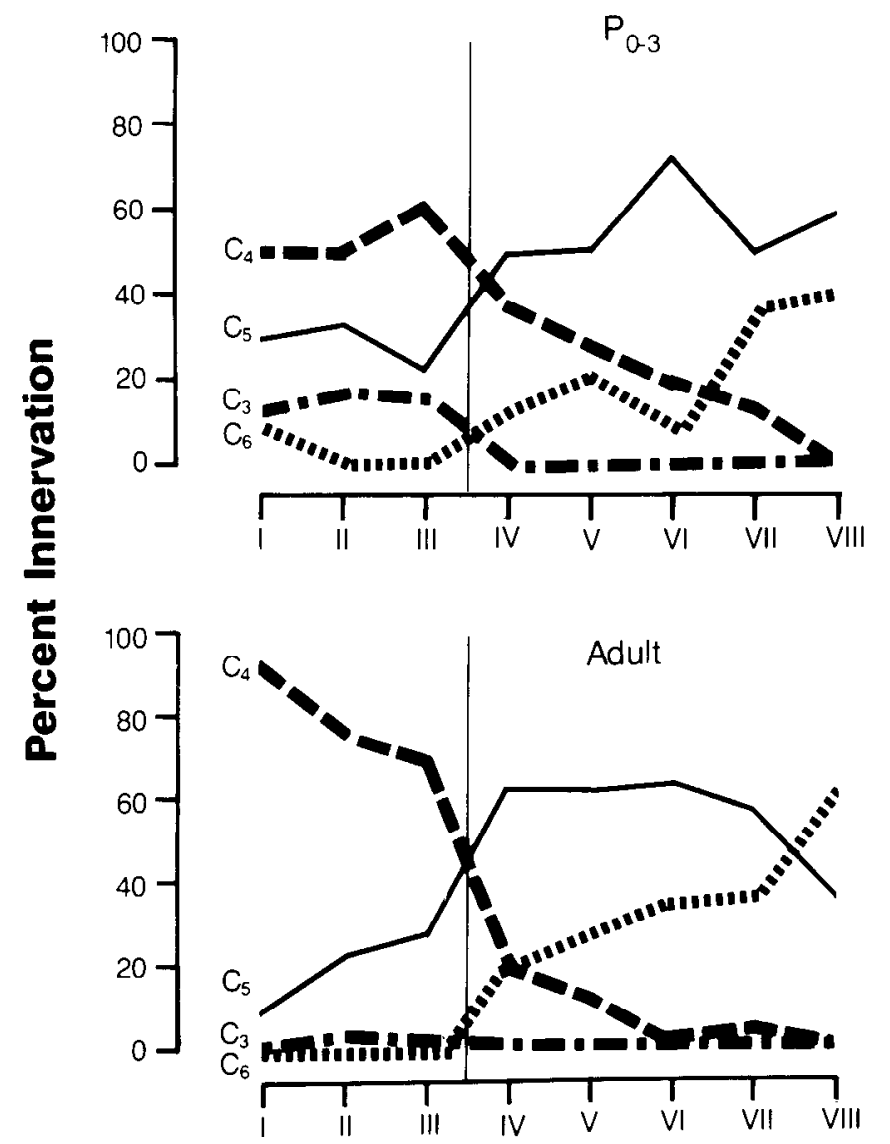

Muscle Sector

Figure 6. Topographic mapping of ventral roots onto the diaphragm in postnatal ( $\mathrm{P} 0-\mathrm{P} 3)$ and adult muscles. For each muscle sector the percentage innervation by $\mathrm{C}_{3}, \mathrm{C}_{4}, \mathrm{C}_{5}$, or $\mathrm{C}_{6}$ is indicated. Vertical line between muscle sectors III and IV represents the bifurcation of the phrenic nerve into rostral and caudal intramuscular branches. Each point represents the mean of 5-10 measurements of synaptic potentials in each of 8 sectors in 4 (neonate) or 10 (adult) muscles.

pattern seen in adult diaphragm muscles, but rather is enhanced and sharpened with subsequent postnatal development.

\section{Discussion}

The most important observation we have made is that the topographic pattern in some muscles is already well established at birth, and, in the serratus at least, it is present a few days prior to birth. Postnatal synaptic rearrangement sharpens this topography toward the adult pattern.

At this point it is important to distinguish between 2 terms used to describe subdivisions of muscle. A fundamental difference may exist between "sectors" in muscle and "compartments." We have defined sectors as anatomically distinct subdivisions within a muscle, wherein each is associated with a different segmental structure, usually a rib. A "compartment" of muscle as described by English and Letbetter (1982a) is a structural and functional unit innervated by a primary branch of a nerve and containing a unique population of motor units. The prototypic compartmentalized muscle is the lateral gastrocnemius of the cat and rat. The extensor digitorum longus (EDL) and intercostal muscles are also compartmentalized (Dennis et al.; 1981; Balice-Gordon and Thompson, 1988b).
Each compartment receives a primary branch of the nerve and is the exclusive territory of a group of motor units. Neuromuscular compartments are already established at birth with little error in misdirected axons (Dennis et al., 1981; Donahue and English, 1987; Balice-Gordon and Thompson, 1988b; Bennett and Ho, 1988). Within a compartment, however, orderly mapping is established only after postnatal synaptic rearrangement has taken place (Bennett and Ho, 1988).

Our observations on muscle sectors cannot be directly compared with those of the above-noted studies until the distribution of individual motor units is precisely mapped on the surfaces of the diaphragm and serratus. Nevertheless, the phrenic nerve does bifurcate into 2 primary branches as it enters the costal portion of the diaphragm, one branch projecting rostrally and the other caudally. Each of these primary branches distributes topographically in adult muscles (Laskowski and Sanes, 1987a). Whether individual motor units restrict their projections to only one of these primary branches in the diaphragm is yet to be determined. For the diaphragm and possibly the serratus, the "sectors" we describe may be subdivisions of compartments. Krnjević and Miledi (1958) demonstrated that single motor units in the diaphragm extend over several millimeters, which would exceed the width of all but the largest sectors. This suggests that sectors in the diaphragm are subdivisions of a compartment whose spatial limits remain to be determined. Whether or not the diaphragm and serratus are eventually found to be composed of a single neuromuscular compartment or more than one does not diminish our fundamental observation, namely, that in these muscles specific connections are established between nerve and muscle prior to birth and prior to postnatal synaptic rearrangment.

The role of postnatal changes in establishing topography remains to be clarified. Postnatal synaptic rearrangement includes the elimination of some nerve terminals coincident to a reduction in motor unit size (Brown et al., 1976; Thompson, 1983; Bennett and Lavidis, 1984a, b; Bennett et al., 1986). At the same time, in some limb muscles new muscle fibers are formed from myotube clusters (Ontell and Dunn, 1978; Betz et al., 1979), and presumably these new fibers become functionally innervated. Thus, postnatal synaptic rearrangment involves both generative and regressive processes. The contribution of synaptic rearrangement toward eventual neuromuscular topography seems to vary among muscles studied. In several limb muscles, postnatal development plays a major role in shaping topography (Brown and Booth, 1983a, b; Bennett and Lavidis, 1987a, b; Bennett and Ho, 1988). The 2 trunk muscles described in this report demonstrate that an ordered map of motoneurons onto their surfaces already exists at birth. Postnatal synaptic rearrangement then plays a less significant role in these muscles. This apparent discrepancy may be resolved once motor unit distributions for each of these muscles have been determined. Nevertheless, even in the diaphragm and serratus, postnatal processes do contribute to the adult pattern of innervation. In the serratus, the greater postnatal change occurs in caudal sectors. During the first 4 weeks after birth, the influence of $\mathrm{C}_{6}$ is reduced and that of $\mathrm{C}_{7}$ is increased in this portion of the muscle. This sharpening of topography emerges at the same time that motor unit size is decreasing. Also in the serratus, $\mathrm{C}_{7}$ innervates a higher proportion of fibers in adult than neonatal muscle. One explanation for this may lie in the formation of new muscle fibers during the postnatal period, although this is yet to be shown in the serratus. The number of new fibers may range 
from $10 \%$ in the EDL (Balice-Gordon and Thompson, 1988a) to nearly a doubling in rat lumbrical and lateral gastrocnemius muscles within the first 2 weeks after birth (Betz et al., 1979; Bennett et al., 1986). If newly formed muscle fibers have a preference for axons according to segment of origin, we would expect to see a more specific neuromuscular map in adults than neonates.

An important concern is whether the sharpening of topography during the period of postnatal synaptic rearrangement results from a selective removal of one or another ventral root or by a random process. Selective removal of one ventral root was observed in the gluteus (Brown and Booth, 1983a, b) and several limb muscles (Bennett and Lavidis, 1984a, b). In the soleus, one report claims that selective elimination of one ventral root hclps to cstablish topography (Miyata and Yoshioka, 1980); however, this has been disputed by evidence suggesting that elimination of redundant terminals is a random process (Gordon and Van Essen, 1983; Thompson, 1983; Balice-Gordon and Thompson, 1988a). Our results do not favor either selective or random removal of the influence of either root in the serratus or the diaphragm. The topography we found in caudal sectors of the serratus could be explained by an equal reduction of motor unit size in both $\mathrm{C}_{6}$ and $\mathrm{C}_{7}$ ventral roots. In this case, as the motor unit size of $\mathrm{C}_{6}$ neurons is reduced, its influence on caudal sectors would be lessened and, as a result, $\mathrm{C}$, would emerge as the dominant root. Alternatively, motor units from $C_{6}$ but not $C_{7}$ might remove their influence selectively from caudal sectors. The same can be said of our observations of the diaphragm. While the influence of $\mathrm{C}_{3}$ is all but lost during postnatal periods, this is not evidence for selective removal. As motor unit size decreases, the small influence of $\mathrm{C}_{3}$ in rostral sectors would be even further reduced. Similarly, in caudal sectors the reduction in motor unit size in $\mathrm{C}_{4}, \mathrm{C}_{5}$, and $\mathrm{C}_{6}$ could also account for the resultant pattern.

In this study, entire ventral roots were stimulated in order to define the development of neuromuscular topography. For the serratus, this meant that the projection of only 2 roots could be evaluated. Ideally, subdivisions of these roots should be stimulated to reveal a smoother map, but this was not technically possible. It is all the more remarkable, therefore, that even this rather coarse estimate was able to detect a rostrocaudal map before birth. If the map can be detected with this level of analysis, it suggests that if it were possible to stimulate small subsets of axons, the topography might be even more apparent. Admittedly, our technique misses some of the subtleties of topography. Nevertheless, it does demonstrate that a topographic map exists at birth. The diaphragm is more revealing in this regard because it is innervated by 4 ventral roots. This muscle also expresses neuromuscular topography at birth. Each ventral root shows a clear territorial preference, which, like the adult, is determined by the bifurcation of the phrenic nerve into rostral or caudal branches (Fig. 6, vertical lines).

In evaluating potential mechanisms that might be responsible for the establishment of topography, attention must be directed toward events occurring in the embryo. A significant change occurring late in embryonic development is the phenomenon of motoneuron cell death (Lance-Jones, 1982; Harris and McCaig, 1984). If cell death occurs in the serratus motor column as it does in the phrenic (Harris and McCaig, 1984), and if selective, it might influence the establishment of topography in the embryo. We have no data to confirm or deny this mechanism; however, one observation may minimize its importance. Our previous reports on selective reinnervation of adult muscles (Laskowski and Sanes, 1987b, 1988) suggest that a topographic bias can be reestablished by regenerating nerves under conditions where substantial motoneuron loss does not occur (Aldskogius and Thomander, 1986). While selective cell death may shape the pattern of innervation, there is little evidence to suggest that it is a fundamental mechanism in establishing topography.

Another potential mechanism underlying topography may be that growing neurites are positionally matched with synaptic targets, at least in a broad statistical sense. Thus, there may not be a point-to-point specificity, but rather a general rostrocaudal directedness toward appropriate synaptic choices. Such matching, even if it is very coarse in its specificity, implies the presence of cues that must guide growing neurites toward appropriate targets. The existence of such cues has been inferred from several studies of reinnervating neurites in both mammalian (Wigston and Sanes, 1982, 1985; Laskowski and Sanes, 1987b, 1988) and lower vertebrates (Wigston, 1986; Wigston and Kennedy, 1987). The location of topographic cues may reside on muscle, on nerve, or another cell type. The results presented in this report as well as previous publications (Wigston and Sanes, 1982, 1985; Laskowski and Sanes, 1987a, b, 1988) suggest the existence of such cues during development and that they remain, albeit less robustly, through adulthood, thus permitting selective reinnervation.

\section{References}

Aldskogius, H., and L. Thomander (1986) Selective reinnervation of somatotopically appropriate muscles after facial nerve transection and regeneration in the neonatal rat. Brain Res. 375: 126-134.

Balice-Gordon, R. J., and W. J. Thompson (1988a) Synaptic rearrangements and alterations in motor unit properties in neonatal rat extensor digitorum longus muscle. J. Physiol. (Lond.) 398: 191-210.

Balice-Gordon, R. J., and W. J. Thompson (1988b) The organization and development of compartmentalized innervation in rat extensor digitorum longus muscle. J. Physiol. (Lond.) 398: 211-231.

Bennett, M. R. (1983) Development of neuromuscular synapses. Physiol. Rev. 63: 915-1048.

Bennett, M. R., and S. Ho (1988) The formation of topographical maps in developing rat gastrocnemius muscle during synapse elimination. I. Physiol. (Lond.) 396: 471-496.

Bennett, M. R., and N. A. Lavidis (1984a) Segmental motor projections to rat muscles during the loss of polyneuronal innervation. Dev. Brain Res. 13: 1-7.

Bennett, M. R., and N. A. Lavidis (1984b) Development of the topographical projection of motor neurons to a rat muscle accompanies loss of polyneuronal innervation. J. Neurosci. 4: 2204-2212.

Bennett, M. R., and A. Pettigrew (1974) The formation of synapses in striated muscle during development. J. Physiol. (Lond.) 241:515545.

Bennett, M., S. Ho, and N. Lavidis (1986) Competition between segmental nerves at end-plates in rat gastrocnemius muscle during loss of polyneuronal innervation. J. Physiol. (Lond.) 381: 351-376.

Betz, W. J., J. H. Caldwell, and R. R. Ribchester (1979) The size of motor units during postnatal development of rat lumbrical muscle. J. Physiol. (Lond.) 297: 463-478.

Brown, M. C., and C. M. Booth (1983a) Segregation of motor nerves on a segmental basis during synapse elimination in neonatal muscles. Brain Res. 273: 188-190.

Brown, M. C., and C. M. Booth (1983b) Postnatal development of the adult pattern of motor axon distribution in rat muscle. Nature 304: 741-742.

Brown, M. C., J. K. S. Jansen, and D. Van Essen (1976) Polyneuronal innervation of skeletal muscle in newborn rats and its elimination during maturation. J. Physiol. (Lond.) 261: 387-422.

Browne, K. M. (1950) The spatial distribution of segmental nerves to striate musculature of the hindlimb of the rat. J. Comp. Neurol. 93: 441-455. 
Dennis, M. J., L. Ziskind-Conhaim, and A. J. Harris (1981) Devel opment of neuromuscular junctions in rat embryos. Dev. Biol. 81 , 266-279.

Donahue, S. P., and A. W. English (1987) The role of synapse elimination in the establishment of neuromuscular compartments. Dev. Biol. 124: 481-489.

English, A. W., and W. D. Letbetter (1982a) Anatomy and innervation patterns of cat lateral gastrocnemius and plantaris muscles. Am. J. Anat. 164: 67-77.

English, A. W., and W. D. Letbetter (1982b) A histochemical analysis of identified compartments in cat lateral gastrocnemius muscle. Anat. Rec. $204: 123-130$

English, A. W., and O. I. Weeks (1984) Compartmentalization of single muscle units in cat lateral gastrocnemius. Exp. Brain Res. 56: 361368.

Gordon, H., and D. C. Van Essen (1983) The relation of neuromuscular synapse elimination to spinal position of rabbit and rat soleus motoneurons. J. Physiol. (Lond.) 339: 591-597.

Harris, A. J., and C. D. McCaig (1984) Motoneuron death and motor unit size during embryonic development of the rat. J. Neurosci. 4 : 13-24.

Krnjević, K., and R. Miledi (1958) Motor units in the rat diaphragm. J. Physiol. (Lond.) 140: 427-439.

Lance-Jones, C. (1982) Motoneuron cell death in the developing lumbar spinal cord of the mouse. Dev. Brain Res. 4: 473-479.

Laskowski, M. B., and J. R. Sanes (1987a) Topographic mapping of motor pools onto skeletal muscles. J. Neurosci. 7: 252-260.

Laskowski, M. B., and J. R. Sanes (1987b) Topographically selective reinnervation of adult mammalian muscles. Soc. Neurosci. Abstr. 13: 1422.

Laskowski, M. B., and J. R. Sanes (1988) Topographically selective reinnervation of adult mammalian skeletal muscles. J. Neurosci. 8 ; 3094-3099.

Lichtman, J. W., and D. Purves (1980) The elimination of redundant preganaglionic innervation to hamster sympathetic ganglionic cells in early postnatal life. J. Physiol. (Lond.) 301: 213-228.
Miyata, Y., and K. Yoshioka (1980) Selective elimination of motor nerve terminals in the rat soleus muscle during development. J. Physiol. (Lond.) 309: 631-646.

Ontell, M. A., and R. F. Dunn (1978) Neonatal muscle growth: A quantitative study. Am. J. Anat. 152: 539-556.

Redfern, P. A. (1970) Neuromuscular transmission in newborn rats. J. Physiol. (Lond.) 209: 701-709.

Sherrington, C. S. (1892) Notes on the arrangement of some motor fibers in the lumbo-sacral plexus. J. Physiol. (Lond.) 13: 621-772.

Soha, J. M., C. Yo, and D. Van Essen (1987) Synapse elimination by fiber type and maturational state in rabbit soleus muscle. Dev. Biol. 123: 136-144.

Swett, J. S., E. Eldred, and J. S. Buchwald (1970) Somatotopic cordto-muscle relations in efferent innervation of cat gastrocnemius. Am. J. Physiol. 219: 762-766.

Thompson, W. J. (1983) Lack of segmental selectivity in elimination of synapses from soleus muscle of newborn rats. J. Physiol. (Lond.) 335: 343-352.

Weeks, O. I., and A. W. English (1985) Compartmentalization of the cat lateral gastrocnemius motor nucleus. J. Comp. Neurol. 235: 255267.

Wigston, D. J. (1986) Selective innervation of transplanted limb muscles by regenerating motor axons in the axolotl. J. Neurosci. 6: 27572763.

Wigston, D. J., and P. R. Kennedy (1987) Selective reinnervation of transplanted muscles by their original motoneurons in the axolotl. J. Neurosci. 7: 1857-1865.

Wigston, D. J., and J. R. Sanes (1982) Selective reinnervation of adult mammalian muscle by axons from different segmental levels. Nature 229: 464-467.

Wigston, D. J., and J. R. Sanes (1985) Selective reinnervation of intercostal muscles transplanted from different segmental levels to a common site. J. Neurosci. 5: 1208-1221. 